\title{
SHORT-TERM HYDRO-THERMAL DISPATCH DETAILED MODEL AND SOLUTIONS
}

\author{
G.X. Luo* H. Habibollahzadeh* $\quad$ A. Semlyen \\ Department of Electrical Engineering \\ University of Toronto \\ Toronto, Ontario, Canada \\ * At present with Ontario Hydro
}

Keywords:Hydro-Thermal Dispatch,Constrained Optimization,Optimal Power Flow,Network Flow Programming

\begin{abstract}
The Short-Term Hydro-Thermal Dispatch approach of this paper has the capability of taking into account the following effects: coupling of cascaded multichained reservoirs, water time delays, reservoir head variations, Load Flow and other constraints due to security and environmental considerations. The problem is decomposed into a hydro and a thermal subproblem which are then solved iteratively. An effective adjustment has been proposed to take into account the nonlinear relation between the two subproblems to speed up the convergence of the iterative process. In this adjustment, as well as in solving the thermal subproblem, Equations of Coordination and Optimal Power Flow are combined for better computational efficiency. Numerical examples are also included in the paper to demonstrate the advantages of the new approach.
\end{abstract}

\section{INTRODUCTION}

The Hydro-Thermal Dispatch problem was solved in two stages in earlier years. The Hydro problem was solved at the first stage to define the hydro production pattern. The second stage was the thermal production dispatch based on the decision of the first stage. Since the Hydro problem and the Thermal problem were solved separately, this two stage approach introduced incoherency between the two subproblems. Therefore, the solution was not optimal. Furthermore, the second stage might not have a feasible solution even thought the overall problem is feasible. This can particularly happen in systems with high share of hydraulic production.

As an improvement, the Short-Term Hydro-Thermal Dispatch (STHTD) problem was solved by employing simplified models both in the hydro subsystem and the thermal subsystem (which contains the transmission network) ${ }^{[1]-[6],(18]}$. These include methods of neglecting in the hydro subsystem one or more of the following effects: couplings of cascaded reservoirs, reservoir head variations and water time delays, and on the thermal subsystem side, of using lossless line models or a loss formula model instead of an Optimal Power Flow (OPF). Although the results are useful under specific circumstances, these methods have the common drawback that their solutions are not guaranteed to be feasible. This is so because some constraints associated with more detailed models have not been taken into account.

To assure that the final solution is feasible, detailed models must be used both in the hydro and the thermal subsystems to formulate the STHTD. Besides, efficient solution methods must be sought because of the high dimension of the STHTD with such detailed models. One of the successful techniques is to decompose the overall problem into two sub-

89 WM 169 -4 PWRS A paper recommended and approved by the IEEE Power System Engineering Committee of the IEEE Power Engineering Society for presentation at the IEEE/PES 1989 Winter Meeting, New York, New York, January 29 - February 3, 1989. Manuscript submitted February 1, 1988; made available for printing December $21,1988$. problems: a hydro subproblem and a thermal subproblem, as in references [7]-[9]. Since these two subproblems are solved iteratively, the solution technique of each subproblem can be improved separately.

Recently, Network Flow Programming has been used to solve the detailed hydro subproblem. It is the most powerful approach in comparison with other methods for solving hydro dispatch problems. Some of the relevant references are [5], [6], [17], [23] and [24].

The solution of the thermal subproblem including OPF has been presented in references [8]-[10]. These results represent the current thrust of research in the area. However, further improvement should be sought to overcome the following drawbacks encountered:

(1) Complicated procedure in constructing a hydro tube ${ }^{[8]}$;

(2) Tedious process of breaking the hydro subproblem into a great number of one dimensional optimization problems ${ }^{[9]}$;

(3) Lack of capability to handle transmission line limits ${ }^{[10]}$.

The work of this paper attempts to give a complete formulation and efficient solution of the Short-Term Hydro-Thermal Dispatch problem. The formulation of the problem includes the head variations, the effect of cascaded multichained reservoirs and the time delays of water flow in the hydro system, and also the effects imposed by the load flow equations and the security constraints. The solution method includes most of the advantages of the existing work in the area and avoids its shortcomings.

The problem examined here is based on the assumption that the unit commitment ${ }^{[17]}$ has been solved separately so that the up-down schedule of the thermal generators is predefined before the problem is being solved.

Based on the above considerations, a decomposition scheme ${ }^{[7]-[9]}$ is adopted. Not only has it been successfully used by others but it also can be rationally derived by mathematical considerations. In addition, Network Flow Programming ${ }^{[11],[6]}$ has been implemented to assure the fast and accurate solution of the hydro subproblem. To speed up the process, the thermal subproblem is solved by a combination of Equations of Coordination (EOC) and OPF. Instead of constructing a hydro tube as in [8] and performing a tedious search as in [9], a line search between each two iterations is performed to improve the thermal generation objective.

The formulation and the solution method proposed in this paper have the following advantages and disadvantages:

Advantage 1: The models for the hydro and thermal systems are detailed and complete. Especially, since the OPF is involved, the final solution is not only more accurate but also feasible.

Advantage 2: The application of Network Flow Programming to the hydro subproblem provides fast and accurate solutions. Thus the tedious process of $[8]$ is avoided.

Advantage 3: Employing a combination of Equations of Coordination and Optimal Power Flows reduces the number of OPF's being invoked. This results in reduction of computation time.

Advantage 4: Using a line search between each two iterations provides an easy way to get the maximum improvement on the objective which has been also sought in [8] and [9].

Disadvantage: Employing Equations of Coordination certainly introduces error. However, by carefully controlling the occasions of using the Equations of Coordination, the error can be limited to an insignificant level. 


\section{THEORETICAL BACKGROUND}

Problem Formulation

With discretization of the total time horizon into a set of shorter time intervals, the Short-Term Hydro-Thermal Dispatch problem can be mathematically formulated as a nonlinear constrained optimization problem as follows:

$$
\begin{aligned}
& \text { P0: } \quad \text { Min. } \sum_{k=1}^{T} \sum_{i=1}^{N_{t}} C_{i k}\left(P_{i k}\right) \\
& \text { s.t. } F_{j k}=r_{j k} q_{j k}-P_{l j k}-f_{j k}\left(\bar{x}_{t k}\right)=0 \\
& \bar{h}_{t k}\left(\bar{x}_{i k}, P_{i k}\right)=0 \\
& \bar{g}_{t k}\left(x_{i k}, P_{i k}\right) \leq 0 \\
& \bar{h}_{h}\left(\bar{x}_{h}, q_{j k}\right)=0 \\
& \bar{g}_{h}\left(\bar{x}_{k}, q_{j k}\right) \leq 0
\end{aligned}
$$

where

$$
\left\{\begin{array}{l}
i=1,2, \ldots, N_{t} \\
j=1,2, \ldots, N_{h} \\
k=1,2, \ldots, T
\end{array}\right.
$$

and

$P_{i k}$ : active power generation of the $i$ 'th thermal generation unit during the $k$ 'th interval;

$C_{i k}$ : generation cost for $P_{i k}$;

$q_{j k}$ : water discharge for power generation of the $j$ 'th hydro station during the $k^{\prime}$ th hour;

$r_{j k}$ : discharge-power conversion factor for the $j$ 'th hydro station during the $k$ 'th hour,

$P_{l j k}$ : active load drawn from the bus connecting to the $j$ th hydro station, during the $k^{\prime}$ th hour,

$\bar{x}_{t k}$ : variable vector, consisting of all the variables associated with the thermal subsystem except $\boldsymbol{P}_{i k}$;

$f_{j k}$ : active power injection from the bus connected to the $j$ 'th hydro station during the $k^{\prime}$ th hour,

$\bar{x}_{h}$ : variable vector, consisting of all the variables associated with the hydro subsystem except $q_{j k}$;

$N_{t}: \quad$ total number of thermal generation units;

$N_{h}$ : total number of hydro stations;

$T$ : total number of time intervals.

Note that the thermal subsystem includes the transmission network Thus $P_{i k}$ and $\bar{x}_{i k}$ form the complete set of thermal variables - variables associated with the thermal system only.

The variable vector $\bar{x}_{h}$ includes the reservoir contents and the nongeneration discharges such as spillages. Along with $r_{j k}$ and $q_{j k}, \bar{x}_{k}$ constitutes the complete set of hydro variables.

The objective of problem $P 0$ represents the total generation cost for the entire period. There are three sets of constraints in the problem. The first set given by (1-2) represents the active power balance at the buses to which the hydro stations are connected. The product $r_{j k} q_{j k}$ denotes the hydro generation. The second set of constraints includes (1-3) and (1-4), the thermal equality and inequality constraints. Generally, $h_{i k}$ in (1-3) represents the load flow equations or their equivalents. The bounds on the thermal variables, the security constraints and the other inequality constraints imposed by special considerations, fall into the subset of $\bar{g}_{t k}$ of (1-4). The third set of constraints, those in (1-5) and (1-6), are the hydro constraints. In (1-5), the equality constraints $\bar{h}_{h}$ represent the water flow balance. Inequalities $\bar{g}_{h}$ in (1-6) represent the bounds on the hydro variables due to physical and operational limitations.

\section{Application of Decomposition}

Problem P0 can be solved by any of the applicable techniques. No matter what kind of method is used, we are facing here a very high dimensional problem. For such a large scale optimization problem, decomposition techniques are very important and beneficial for savings in computer storage and CPU time ${ }^{[7],[8]}$
To examine the necessary conditions for a solution to problem $\mathbf{P 0}$ The Kuhn-Tucker condition ${ }^{[12]}$ for PO is given by (2) and (3), jointly. The complementary condition can be taken into account implicitly in (2) and (3). Then, the inactive inequality constraints and their associated Lagrangian multipliers will not be considered.

$$
\left\{\begin{array}{l}
\sum_{k=1}^{T} \sum_{i=1}^{N_{t}} \frac{d C_{i k}}{d P_{i k}}+\bar{\lambda}_{i k}^{T} \frac{\partial \bar{h}_{i k}}{\partial P_{i k}}+\bar{\mu}_{i k}^{T} \frac{\partial \bar{g}_{i k}}{\partial P_{i k}}=0 \\
\sum_{j, k} \lambda_{j k} \frac{\partial F_{j k}}{\partial \bar{x}_{i k}}+\bar{\lambda}_{i k}^{T} \frac{\partial \bar{h}_{t k}}{\partial \bar{x}_{i k}}+\bar{\mu}_{t k}^{T} \frac{\partial \bar{g}_{t k}}{\partial \bar{x}_{t k}}=0 \\
\bar{F}_{j, k}=0 \\
\bar{h}_{t k}=0 \\
\bar{g}_{t k} \leq 0
\end{array}\right.
$$

$$
\left\{\begin{array}{l}
\sum_{j, k} \lambda_{j k} \frac{\partial F_{j k}}{\partial q_{j k}}+\bar{\lambda}_{h}^{T} \frac{\partial \bar{h}_{h}}{\partial q_{j k}}+\bar{\mu}_{h}^{T} \frac{\partial \bar{g}_{h}}{\partial q_{j k}}=0 \\
\bar{\lambda}_{h}^{T} \frac{\partial \bar{h}_{h}}{\partial \bar{x}_{h}}+\bar{\mu}_{h}^{T} \frac{\partial \bar{g}_{h}}{\partial \bar{x}_{h}}=0 \\
\bar{h}_{h}=0 \\
\bar{g}_{h} \leq 0
\end{array}\right.
$$

Assuming proper convexity of the objective and the constraints of problem PO, the Kuhn-Tucker conditions in Eqn. (2) and in Eqn. (3) give the necessary and sufficient conditions of defining a solution for the problem since they satisfy both the first and second order conditions ${ }^{[12]}$ in optimization theory.

The Kuhn-Tucker conditions given in (2) and (3) can be satisfied by iteratively solving at each time Eqn. (2) and Eqn. (3). This process is equivalent to iteratively solving two optimization subproblems: the thermal subproblem and the hydro subproblem,

P1: (Thermal Subproblem)

$$
\begin{aligned}
\text { Min. } & \sum_{k=1}^{T} \sum_{i=1}^{N_{t}} C_{i k}\left(P_{i k}\right) \\
\text { s.t. } & F_{j k}=r_{j k} q_{j k}-P_{l j k}-f_{j k}\left(\bar{x}_{i k}\right)=0 \\
& \bar{h}_{i k}\left(\bar{x}_{i k}, P_{i k}\right)=0 \\
& \bar{g}_{i k}\left(\bar{x}_{i k}, P_{i k}\right) \leq 0
\end{aligned}
$$

P2: (Hydro Subproblem)

$$
\begin{array}{r}
\text { Min. } \sum_{j_{1} k} \lambda_{j k} \cdot r_{j k} \cdot q_{j k} \\
\text { s.t. } \bar{h}_{h}\left(\bar{x}_{h}, q_{j k}\right)=0 \\
\bar{q}_{h}\left(\bar{x}_{h}, q_{j k}\right) \leq 0
\end{array}
$$

The decomposition of $\mathrm{P} 1$ and $\mathrm{P} 2$ from $\mathrm{P} 0$ is accomplished by properly dividing the constraints into the two subproblems and by assigning a carefully constructed objective to the hydro subproblem.

The correctness of decomposing P0 into $\mathrm{P} 1$ and $\mathrm{P} 2$ can be clarified. An easy way is to compare the Kuhn-Tucker conditions for P1 and P2 together, with those for PO. In fact, condition (2) is exactly the KuhnTucker conditions for P1 and condition (3) for P2.

\section{Iteration Algorithm}

The algorithm for solving the thermal subproblem and hydro subproblem iteratively can be summarized, for the $k^{\prime}$ th iteration, as follows.

\section{Algorithm A 1:}

Step (a): With the $r_{j k}$ and $q_{j k}$ obtained from the $(k-1)$ 'th iteration kept constant, $P 1$ is solved to obtain the $\lambda$ 's; 
Step (b): With the $\lambda$ 's from Step (a), solve P2 to obtain the hydro productions in terms of $r$ and $q$;

Step (c): Check the convergence. Stop if convergence has been reached, otherwise go to Step (d);

Step (d): Perform a line search (to be explained later) to obtain the direc tional minima and to determine the associated $r_{j k}$ and $q_{j k}$, and then go back to step (a) to start a new iteration.

The above algorithm is also shown in Figure 1 with the corresponding computational flow chart.

Theoretically, iterations between Problem P1 and Problem P2 should continue until both subproblems give the same $\lambda_{j k}$ 's and $q_{j k}$ 's Practically, this is very time consuming because $\lambda_{j k}$ and $q_{j k}$ comprise many variables. Therefore, a different stopping criterion is applied to speed up the procedure without introducing noticeable errors. This is to stop the process when the improvement on the objective becomes small enough or the objective itself is close enough to its lower bound.

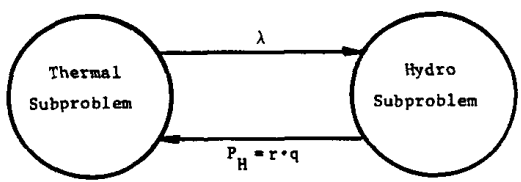

(a) Relation of the two subproblems.

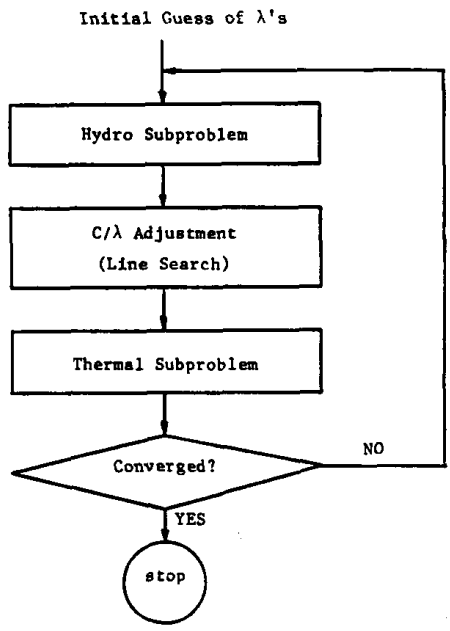

(b) Flow chart of Algorithm A1.

Figure 1 : Iterative algorithm for solving the Short-Term Hydro-Thermal Dispatch problem.

So far, Step (d) in Algorithm Al and its corresponding block named ' $\lambda$ Adjustment' in Figure 1 have not been discussed. The importance and the implementation of this block will be explained later.

\section{THERMAL SUBPROBLEM}

Noting that the thermal subproblem is a separable problem ${ }^{[8]}$ with respect to time, it can be further decomposed into a set of optimal dispatch problems. A solution based on a detailed model of the thermal subsystem is the $\mathrm{OPF}^{[13],[14]}$

In general, any OPF can be plugged in as a module to fulfil the task. Minor changes in an existing module might be needed to meet the following requirements:

(1) At each bus connecting to a hydro station, the active power production at that bus will no longer be a control variable but predefined by the solution of the hydro subproblem.
(2) In the output, the bus incremental cost of each bus connecting to a hydro station has to be evaluated. This is the dual variable of the power balance constraint at that bus. This extra work provides the $\lambda$ 's needed by the hydro subproblem.

The OPF implemented in this work is based on a branch-oriented formulation and Successive Linear Programming solution technique. Details of this module can be found in References [16] and [19].

\section{HYDRO SUBPROBLEM}

\section{Hydro power production}

The power production from a hydro station is a function of turbine discharge and effective plant head. The power production at a constant head versus discharge can be given approximately by a piecewise linear function as shown in Figure 2. This defines the relation between $P$ and $q$

$$
P_{H}=P_{H}^{m}+\sum_{l=1}^{L} r_{l} q_{l}
$$

The solid curve in Figure 2 corresponds to a certain given head. In practice, the head is varying between its upper and lower bounds. Therefore, the curves corresponding to these upper and lower bounds are given and are shown in Figure 2 (the dashed lines). The power curve corresponding to the intermediate heads is produced by interpolation of these extreme curves.

In Figure 2, the $r$ 's are the slopes of the line segments. Therefore, the discharge-power conversion factors $r$ are head dependent.

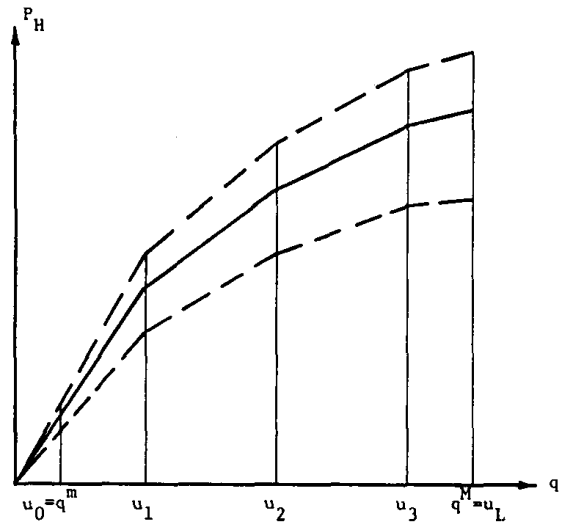

Figure 2: Piecewise linear approximation of hydro production curve.

\section{Reservoir dynamics}

The water flow balance must be met during each hour for every reservoir. This can be mathematically represented by a difference equation:

$$
\begin{gathered}
v(j, k+1)-v(j, k)+\sum_{l=1}^{L_{j}} q_{l}(j, k)+s(j, k) \\
-\sum_{m} \sum_{l=1}^{L_{m}} q_{l}\left(m, k-\tau_{m j}\right)-\sum_{m} s\left(m, k-\tau_{m j}\right)-w(j, k)=0 \\
(j \in J, k \in K)
\end{gathered}
$$

where

$\Sigma: \quad$ sum of discharges from upstream reservoir(s)

$J$ : $\quad$ set of indices for the hydro elements in the system

$K$ : set of indices for the periods in the optimization

$v(j, k)$ : the content of reservoir $j$ at the beginning of hour $k$

$q_{t}(j, k)$ : the $t$ th component of turbine discharge of reservoir $j$ during hour $k$ 
$L_{j}: \quad$ total number of components in turbine discharge of the station coupled to reservoir $J$

$s(j, k)$ : spillage of reservoir $j$ during hour $k$

$\tau_{m j}: \quad$ time delay (in hours) for water to reach reservoir $j$ from its upstream reservoir $m$

$w(j, k)$ : natural inflow to reservoir $j$ during hour $k$.

\section{Head variations}

It has been mentioned that the discharge-power conversion factors are functions of the effective head $H_{e}$ :

$$
r=r\left(H_{e}\right) \text {. }
$$

The effective head is equal to the difference of the forebay elevation $H_{f}$ and the tail race elevation $H_{t}$ of the reservoir

$$
H_{e}=H_{f}-H_{t} \text {. }
$$

Generally, the forebay elevation and the tail race elevation depend on $q, v$ and $s$ in the station as well as in the upstream and downstream stations. This can be summarized by

$$
\begin{aligned}
& H_{f}=H_{f}(\bar{q}, \bar{s}, \bar{v}) \\
& H_{t}=H_{t}(\bar{q}, \bar{s}, \bar{v})
\end{aligned}
$$

Equation (8) to (11) represent the variation of the effective head in a station. It should be noted that only ( 9 ) is linear, the remaining relations being nonlinear in general. However, equations (10) and (11) are assumed linear (i.e. $H=k v$ ) to guarantee the convexity of the problem. This assumption is quite acceptable in a short-term study since the reservoir head does not normally vary too much during a day.

\section{Complete Hydro Subproblem Formulation and Algorithm}

The complete hydro subproblem, as modeled in the previous sections, is as follows:

$$
\begin{aligned}
\text { P3: } & \text { Max. } \\
& \sum_{k=1}^{T} \sum_{j=1}^{N_{h}}\left(-\lambda_{j k}\right) \cdot\left[\sum_{l=1}^{L_{j}} r_{j k l} \cdot q_{j k l}\right) \\
\text { s.t. } & \bar{h}_{h}(\bar{q}, \bar{s}, \bar{v})=0 \\
& \bar{g}_{h}(\bar{q}, \bar{s}, \bar{v}) \leq 0
\end{aligned}
$$

with the head variations governed by the functions

$$
\begin{aligned}
& r=r\left(H_{e}\right) \\
& H_{e}=H_{f}-H_{t} \\
& H_{f}=H_{f}(\bar{q}, \bar{s}, \bar{v}) \\
& H_{t}=H_{t}(\bar{q}, \bar{s}, \bar{v})
\end{aligned}
$$

In problem $\mathrm{P} 3$, the minimization problem $\mathrm{P} 2$ has been converted to a maximization problem by reversing the sign of the objective. Here $-\lambda_{j k}$ is positive. The equality constraints $(12-2)$ were represented in equation (7). The inequality constraints (12-3) include all the bounds on the discharges, spillages and reservoir contents.

For constant reservoir heads, expressions (12-1), (12-2) and (12-3) are enough to define the problem. In this case, since constraints (12-2) and $(12-3)$ have a pure network structure, the problem can be solved by applying a network flow algorithm in a single step. However, head variations have been accounted for in P3. In order to solve P3 completely, extra steps must be taken and iterations are needed. The following is the algorithm for solving the hydro subproblem P3.

(a) Initializations: Initialize $r$ 's based on a guess of $H_{e}$ 's.

(b) Construct a maximization problem consisting of (12-1), (12-2) and (12-3). Solve this problem for $\bar{q}, \bar{s}$ and $\bar{v}$.

(c) Convergency check: Check whether the differences in $\bar{q}, \bar{s}$ and $\bar{v}$ obtained from the last two iterations are within the preset error tolerance. If they are, stop. Otherwise,

(d) Update $r$ 's by substituting the current $\bar{q}, \bar{s}$ and $\bar{v}$ into functions (124), (12-5), (12-6) and (12-7). Go back to step (b).

The algorithm described above has a very fast convergence characteristic. This is because the head variations are generally very small within a day or a week -- the short term Dispatch horizon.

\section{$C / \lambda$ ADJUSTMENT}

Importance of the $C / \boldsymbol{\lambda}$ Adjustment

Oscillations have been noticed if the hydro-thermal problem is solved by direct iteration of the hydro and thermal subproblems. The reason for the oscillatory behavior is, on one hand, the nonlinear relation of the discharge $q$ and the bus incremental cost $\lambda$. On the other hand, the input-output $(\lambda-q)$ relation of the hydro subproblem, solved by network flow programming, is linear. An illustration of such oscillations in a simple case andits elimination have been given in the Appendix.

In order to assure that the process is convergent, the oscillations must be eliminated. A special treatment of this problem, called $C / \lambda$ Adjustment, which inserts a line search in between two iterations, will be discussed.

The search is performed on the 'line segment' connecting the previous and current solutions of the Hydro Subproblem (water dispatch). For each particular point on the 'line' the water dispatch can be defined and also the hydro production in the system. Based on this, a Thermal Subproblem is solved to check the feasibility and/or to find the generation cost. In our approach, a Fibonacci search is used to find the point corresponding to the minimum generation cost of the line search. Let $\bar{P}_{p}$ and $P_{c}$ be the previous and current hydro production vectors and $k$ a coefficient between 0 and 1 . The algorithm for the line search is as follows:

(a) Select $k$ according to the Fibonacci technique ${ }^{[12]}$.

(b) Form the hydro production vector $\bar{P}=\bar{P}_{p}+k\left(\bar{P}_{c}-\bar{P}_{p}\right)$.

(c) Solve the thermal subproblem based on $\bar{P}$.

(d) Check whether the current point is optimal. If not, go back to (a).

The proposed line search seems very simple in principle. However, it is very costly. For each point selected in the line search, we have to solve the thermal subproblem for the thermal generation cost $C$ and for the bus incremental cost $\lambda$ based on the corresponding hydro productions. This will involve solving a set of OPF's each of which is costly to perform. Moreover, repeating the above procedure is normally needed to complete one line search.

However, the line search need not be highly accurate. In fact, after each line search, the solution process will continue by another iteration unless convergence has been reached.

Due to the above considerations, employing a combination of Equations of Coordination ${ }^{[15]}$ (EOC) and OPF in the line search has been proposed and implemented. To keep the approximation acceptable, OPF is used whenever performing an OPF is essential.

It is well known that the difference between the EOC and OPF is that the former do not take into account the voltages and reactive generations as control variables. Besides, they give the economic dispatch without considering the following constraints: the generator voltage limits, the reactive generation $\left(Q_{g}\right)$ limits and the line current $\left(I_{l}\right)$ limits.

Also, it has been shown in the past ${ }^{[15]}$ that the power dispatch given by the EOC is still quite close to the accurate result produced by an $O P F$, if no limits on $Q_{g}$ and $I_{l}$ are violated.

However, the situation will be totally different if limits on the reactive generations $Q_{g}$ or on the line curnents $I_{l}$ are violated. In such cases, the generation costs $C$ and the bus incremental costs $\lambda$ evaluated by the EOC are no longer correct. This is so because the resultant dispatch is no longer optimal with respect to the $Q_{g}$ and $I_{l}$ limits. Then, instead of calling the EOC, OPF should be called to yield a true optimal dispatch as well as the related $C$ and $\lambda$ 's.

The conclusions drawn from the above discussions can be summarized as follows: In the line search, OPF can be fairly well approximated by an EOC except in the cases in which the resultant solution from the EOC violates the limits on line current $I_{l}$ or on reactive generations $Q_{g}$.

\section{Computational Flow Chart}

The task of the $C / \lambda$ Adjustment is to perform a line search to determine the point at which the cost is minimum. The main concern of the $C / \lambda$ Adjustment is how to efficiently evaluate the thermal generation cost and the bus incremental costs for each particular point during the line search. The basic strategy is to use EOC as much as possible unless when performing an OPF is essential. The procedure of estimating $C$ and $\lambda$ 's for a single time interval will be explained later. Overall 
estimations for the entire horizon on $C$ and $\lambda$ 's can be made by repeating this procedure.

The procedure of estimating $C$ and $\lambda$ 's for a single time interval first invokes EOC to determine the thermal generation dispatch. After this has been done, a load flow is performed with the following specified quantities:

(1) hydro productions determined by the hydro subproblem and the point selected during the line search;

(2) thermal productions determined by the EOC, except the one for the slack generator,

(3) reasonable preset voltages for all $P-V$ buses and the slack bus.

The preset voltages for the load flow needs to be carefully chosen not to violate their limits. To see whether the EOC is satisfactory, the results given by the load flow should be checked against the complete set of the constraints in OPF. If the solution from the load flow calculation is feasible, the results produced by the EOC are accepted. Otherwise, an OPF is performed to evaluate the true $C$ and $\lambda$ 's.

There is not much work involved in the feasibility check on the load flow results just mentioned. Note that most of the constraints in OPF have been automatically satisfied by the load flow solution. These include all the equality constraints, the voltage limits and the active generation limits. As a result of this, only the line flow limits and the reactive generation limits need to be checked.

Figure 3 shows the computational flow chart of the $C / \lambda$ Adjustment. In the figure, only the procedure of estimating the $C$ and $\lambda$ 's for a single time interval has been shown. The reader should notice that the loop for the time intervals and the loop required by the line search have been omitted.

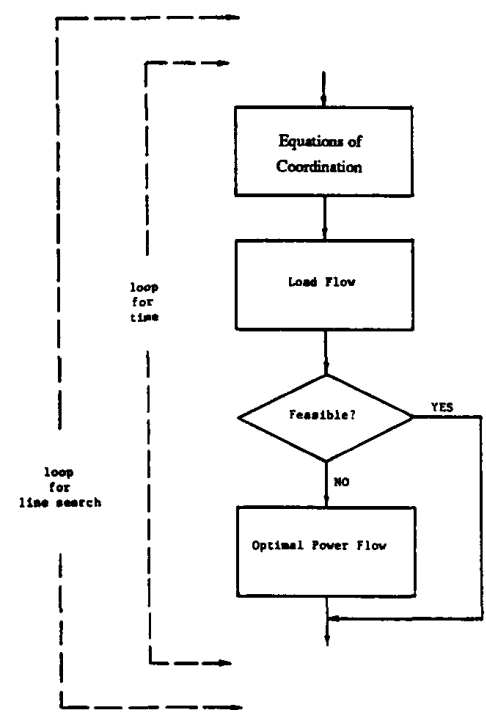

Figure 3: Flow chart of the $C / \lambda$ Adjustment.

\section{METHODOLOGIES}

According to the existing literature, there are different techniques for solving the thermal subproblem according to the degree of detail in the thermal subsystem modeling. Each technique is based on one of the following:

(1) lossless model using priority list only ${ }^{[5-[6]}$;

(2) loss formula model using $\mathrm{EOC}^{[1\}-[4]}$

(3) complete model using $\mathrm{OPF}^{[8\}-[10]}$.

Techniques for solving the thermal subproblem can also be used in the line search. By employing different techniques for performing the line search and/or for solving the thermal subproblem, four different methods are proposed, based on the flow chart shown in Fig. 1, to solve the Short-Term Hydro-Thermal Dispatch in this work. This has been shown in Figure 4.

Method 1 employs only the priority list to solve the thermal subproblem. The same principle is applied to the line search. In this method, the thermal subproblem can be solved through the following steps: (1) Find the economic thermal generation dispatch along the priority list, (2) Calculate the thermal generation cost of the system: (3) Pick up the cost coefficient of the last committed segment in the priority list and take this cost as the bus incremental cost for every bus.

\begin{tabular}{|c||c|c|c|c|}
\hline & 1 & 2 & 3 & 4 \\
\hline \hline $\begin{array}{c}\text { Line } \\
\text { Search }\end{array}$ & $\begin{array}{c}\text { Priority } \\
\text { List }\end{array}$ & EOC & $\begin{array}{c}\text { EOC } \\
+ \text { OPF }\end{array}$ & $\begin{array}{c}\text { EOC } \\
+ \text { OPF }\end{array}$ \\
\hline $\begin{array}{c}\text { Thermal } \\
\text { Subproblem }\end{array}$ & $\begin{array}{c}\text { Priority } \\
\text { List }\end{array}$ & EOC & OPF & $\begin{array}{c}\text { EOC } \\
+ \text { OPF }\end{array}$ \\
\hline
\end{tabular}

Figure 4: Method descriptions.

Method 1 ignores the systems' losses and does not distinguish the bus incremental costs. In fact it has ignored the whole transmission network. The main advantage of Method 1 is its great simplicity. However, since the thermal subsystem has been over-simplified, the errors are expected to be large in the solutions given by Method 1. Moreover, there is no guarantee for its solutions to be feasible.

Method 2 uses a loss formula to represent the thermal subsystem. It provides the possibility to take into account the system losses and to distinguish the bus incremental costs. Since the bus incremental costs serve as the weighting factors to guide the optimal water allocation in the hydro subsystem, better hydro power production dispatch can be obtained in Method 2. Consequently, it yields a better dispatch of the thermal generations. However, Method 2 still cannot avoid leading to infeasible solutions due to the limitations of the Equations of Coordination .

In Method 3, the $C / \lambda$ Adjustment, a combination of EOC and OPF is used in the line search. As to the thermal subproblem, only OPF is employed. In this method, models are complete for the whole hydrothermal system and hence there are no assumptions involved. Therefore, solutions given by Method 3 will be treated as standard.

Method 4 is a modified version of Method 3. The modification is to extend the combination of EOC and OPF to solve the thermal subproblem as well. This method has the same advantages as Method 3 but certainly reduces the total number of OPFs performed in the whole process. Great savings on CPU time are expected in this method.

Since the EOC is involved in solving the thermal subproblem, errors will be introduced in Method 4. However, the errors are expected to be small and acceptable. This is because the only source of errors comes from the approximation of using Equations of Coordination to replace OPF in, and only in, the cases when OPF is not essential.

All four methods proposed above have been tested. Computational results and comments on their performance will be presented next.

\section{NUMERICAL TESTS}

Results from the numerical tests of the proposed hydro subproblem, the accuracy in estimating $C$ and $\lambda$ by EOC and Short-Term Hydro-Thermal Dispatch will be presented. All tests were run on a Honeywell DPS-8 computer. Shorter times could be expected on a VAX minicomputer.

\section{Tests on the Hydro Subproblem}

Features of the hydro subproblem mentioned previously are all considered in the tests. The total time horizon is set to 24 hours, which is discretized into subintervals of one hour. Due to the fact that the optimization horizon is short and the reservoir head cannot vary much, head variation versus reservoir content is assumed linear.

Results of the tests in the three hydro systems used in this study are summarized in Figure 5. In the figure, the size of the problems and the timing of the computations have been listed. The CPU time for 'preparations' is required for arranging the problem to have a network structure. 


\begin{tabular}{|l|c|c|c|}
\hline \multicolumn{1}{|c|}{ Description } & System 1 & System 2 & System 3 \\
\hline No. of rivers & 1 & 2 & 3 \\
\hline No. of stations & 8 & 16 & 28 \\
\hline No. of reservoirs & 6 & 13 & 22 \\
\hline No. of variables & 1146 & 2363 & 3611 \\
\hline No. of constraints & 301 & 551 & 926 \\
\hline CPU for preparations (s) & 2.6 & 4.6 & 6.5 \\
\hline CPU for iteration 1 (s) & 6.7 & 18.3 & 37.8 \\
\hline CPU for other iterations (s) & 1.1 & 4.1 & 5.0 \\
\hline $\begin{array}{l}\text { No. of extra head } \\
\text { iterations }\end{array}$ & 1 & 1 & 2 \\
\hline Total CPU(s) & 9.4 & 25.0 & 54.3 \\
\hline
\end{tabular}

Figure 5: Summary of the tests in the hydro subproblem.

\section{Tests on the accuracy of EOC}

The purpose of this test is to verify that the Equations of Coordination can give results of generation cost $C$ and bus incremental costs $\lambda$ close enough to those obtained from an OPF. This is important since it provides a strong support to our proposal of employing the EOC to replace the OPF in the line search and/or in solving the thermal subproblem.

Tests are performed by directly comparing the resultant $C$ and $\lambda$ 's obtained from the EOC to those from an OPF for the same thermal subproblem. Due to the limitations of the EOC discussed before, comparisons are only taken in the cases in which the operating situation of the thermal system satisfies the condition required by the EOC.

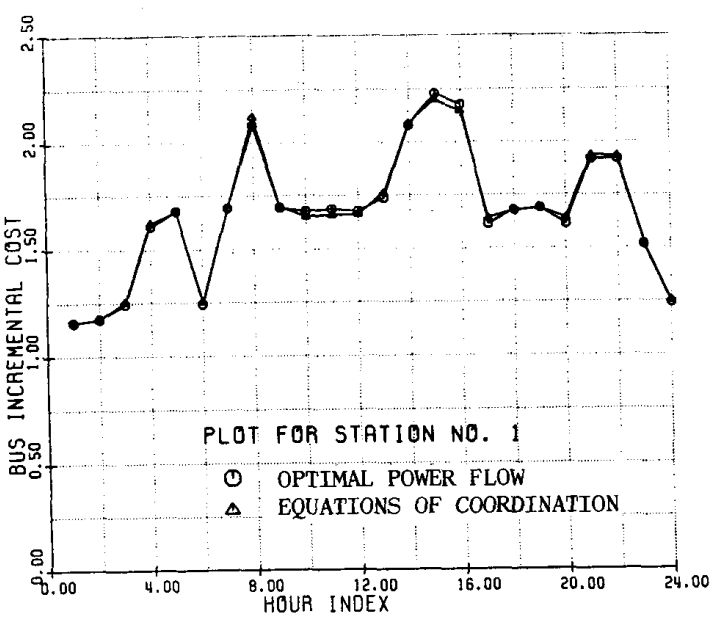

(a) Comparison in $\lambda$ 's.

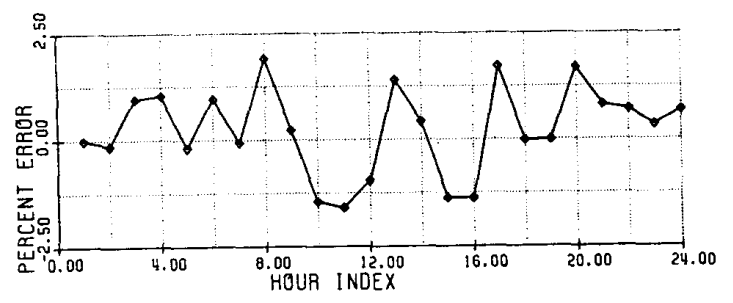

(b) Relative errors on $\lambda$ 's.

Figure 6: Comparison in $\lambda$ 's for a bus.

Figure 6 shows the results of the comparison of $\lambda$ 's at a bus over a 24 hour horizon, for a particular problem in a 14-bus system. These results are obtained from one of the iterations when solving the ShortTerm Hydro-Thermal Dispatch. In Figure 6(a), the bus incremental cost for the bus connecting to Station 1 is presented by two curves: one from the EOC and another from the OPF. Note that the two curves are very close. The errors produced by the $C / \lambda$ estimator are within $\pm 2.5 \%$ when the results from OPF are taken as standard. This relative error has been plotted in Figure 6(b).

The same tests are also applied to other buses in the 14-bus system and have been performed in the 39-bus system (see Fig. 11). Results from the tests also show that $\pm 2.5 \%$ are the bounds of the relative errors in $\lambda$ 's.

Total generation cost $C$ is also compared in the same way as that for $\lambda$ 's. Much smaller errors have resulted. According to the tests on both systems, the relative errors in $C$ have never exceeded $\pm 0.06 \%$.

Test on Short-Term Hydro-Thermal Dispatch Solved by the Proposed Methodology

In order to investigate the performance of the proposed methodology, the four different methods discussed in this paper have been tested in two hydro-thermal systems: H-T System A and H-T System B.

\section{.- Tests on H-T System A}

H-T System A consists of 5 thermal generation units, 4 hydro stations. and a 14-bus, 15-line transmission network.

Tests were first performed in a base case, a case the final solution of which does not violate any of the line current limits and/or the reactive generation limits. All four methods discussed have been implemented in this case. Results of interest from the tests have been given in Figure 7.

\begin{tabular}{|l|c|c|c|c|}
\hline \multicolumn{1}{|c|}{ Method No. } & 1 & 2 & 3 & 4 \\
\hline Total generation cost & 243.119 & 228.550 & 228.543 & 228.550 \\
\hline Per cent error in cost ${ }^{(*)}$ & $+6.4 \%$ & $\approx 0$ & 0.0 & $\approx 0$ \\
\hline No. of iterations & 5 & 4 & 4 & 4 \\
\hline No. of OPF performed & 0 & 0 & 48 & 0 \\
\hline Total CPU (min.) & 0.4 & 0.5 & 46 & 1.4 \\
\hline
\end{tabular}

$\left(^{*}\right)$ Taking result of Method 3 as standard.

Figure 7: Test results in base case of H-T System A.

Taking the results produced by Method 3 as the standard, it is shown that Method 1 has a noticeable error in its solution. This is expected since Method 1 has oversimplified the problem. Method 2 should also produce some error. However, such an error does not appear in Figure 7. This is because no line current limits and/or reactive generation limits are violated by the final solution of the problem.

To show the deficiency of Method 2, a case involving some violations on the transmission line current limits was constructed and applied to the tests. By observing the line currents in the final solution of the base case, we have set the current limit on line No. 1 of the system $12.5 \%$ lower than its value in the final solution of the base case, for hours 3-5 only. For convenience, we name this case Case 1. Again, Methods 2, 3 and 4 were performed in Case 1 and their results have been shown in Figure 8. The reason for having dropped Method 1 in the test is because its deficiency has already been shown and we are no longer interested in it.

\begin{tabular}{|l|c|c|c|}
\hline \multicolumn{1}{|c|}{ Method No. } & 2 & 3 & 4 \\
\hline Total generation cost & 230.506 & 228.793 & 228.799 \\
\hline Per cent error in cost & $+0.75 \%$ & 0.0 & $\approx 0$ \\
\hline No. of iterations & 4 & 3 & 3 \\
\hline No. of OPF performed & 0 & 33 & 12 \\
\hline Total CPU (min.) & 0.5 & 32 & 12 \\
\hline
\end{tabular}

Figure 8: Test results in Case 1 of H-T System A.

The total generation cost obtained by Method 2 in Figure 8 is by $0.75 \%$ higher than that in Figure 7 . This is due to the necessary adjustment by the post-procedure (a set of OPFs) which adjusts the final thermal production dispatch given by Method 2 to satisfy the reduced line current limits. It is interesting to note that the final result from Method 2 
does not depend on the current limits but the post-procedure does. As to Method 3, since the new current limits have been taken into account through the OPF, the optimal solution can be found by automatically adjusting the hydro production dispatch to release the binding current limits during the line search in the process. This yields a total generation cost modestly higher than that in the base case, subject to the satisfaction of the reduced current limits.

In comparison with the results produced by Method 3, the total generation cost given by Method 2 with post-procedure is $0.75 \%$ higher. This shows one of the deficiences of Method 2. However, this is not all the shortcomings of Method 2. As we will see later, its main deficiency is that it can lead to an infeasible solution.

By further reducing the current limit for line No. 1 to $25 \%$ lower than that in the final solution of the base case for hours 3-5, another case, named Case 2, is constructed. The same tests as performed in Case 1 were performed in Case 2. Figure 9 presents the results from the tests. In this case, the final solution given by Method 2 becomes infeasible. As to Method 3 and Method 4, feasible results have been obtained and the total generation cost only increases slightly compared to that in Case 1.

As mentioned before, the value of Method 3 consists in its capability to take into account the constraints which Method 2 cannot handle. Due to this, the process in Method 3 is guaranteed to converge to the true feasible optimal solution by searching for the true minima, and at the same time automatically dropping those points which are detected to be infeasible during the line search.

\begin{tabular}{|l|c|c|c|}
\hline Method No. & 2 & 3 & 4 \\
\hline Total generation cost & Solution & 229.060 & 229.092 \\
\cline { 3 - 4 } Per cent error in cost & Infeasible & 0.0 & $\approx 0$ \\
\hline No. of iterations & 4 & 3 & 3 \\
\hline No. of OPF performed & 0 & 39 & 15 \\
\hline Total CPU (min.) & 0.5 & 40 & 16 \\
\hline
\end{tabular}

Figure 9: Test results in Case 2 of H-T System A.

Comparing the results produced by Method 4 to those by Method 3 in all three cases shows that Method 4 is the best. Method 4 yields almost the same results as Method 3 in all cases but significantly reduces the CPU time requirement. This is due to the reduction on the number of OPF performed.

\section{-- Tests on H-T System B}

H-T System B is bigger than H-T System A in size. It consists of 6 thermal generation units, 7 hydro stations which spread along two rivers, and a 39-bus, 46-line transmission network. Figure 10 gives the configuration of its hydro subsystem, and its thermal subsystem is shown in Figure 11.

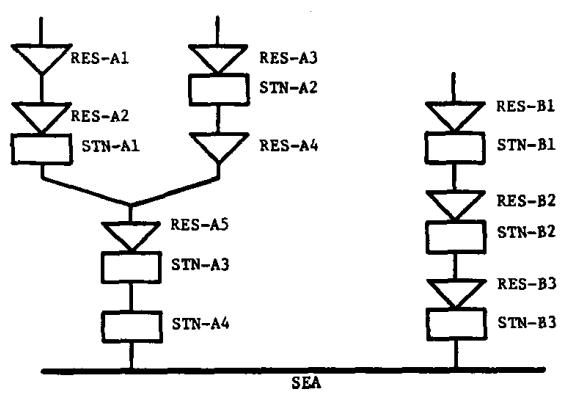

Figure 10: Hydro subsystem in H-T System B.

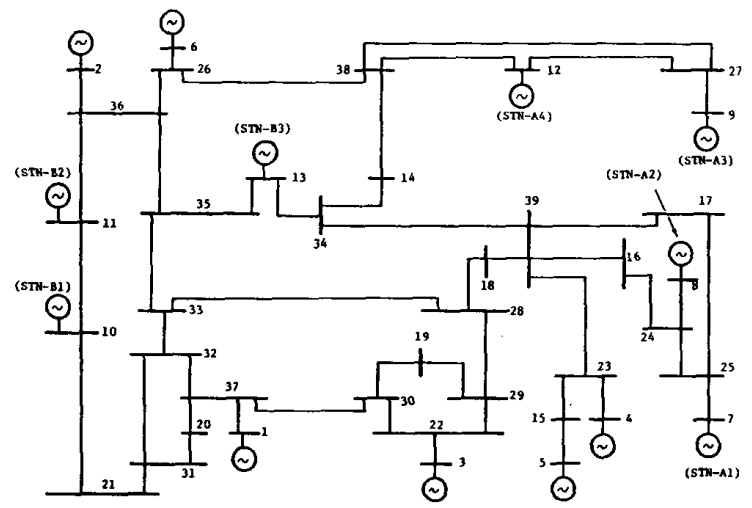

Figure 11: Thermal subsystem in H-T System B.

Similarly to the tests performed in H-T System A, a base case was first chosen for the tests. Results from the tests in the base case are presented in Figure 12. Like the results of the base case in H-T System A, Figure 12 shows again that Method 1 results in a noticeable error but Method 2 remains a good approximation to Methods 3 and 4 .

\begin{tabular}{|l|c|c|c|c|}
\hline Method No. & 1 & 2 & 3 & 4 \\
\hline Total generation cost $^{*}$ & 716.07 & 706.96 & 706.64 & 706.84 \\
\hline Percent error in cost $^{(}$) & $+3.6 \%$ & $\approx 0.0$ & 0 & $\approx 0.0$ \\
\hline No. of iterations & 4 & 3 & 3 & 3 \\
\hline No. of OPF performed & 0 & 0 & 24 & 0 \\
\hline Total CPU (min) & 0.8 & 1.0 & 81 & 5.2 \\
\hline
\end{tabular}

$\left(^{*}\right)$ Taking results of Method 3 as standard.

Figure 12: Test results in base case of H-T System B.

In order to show the deficiency of Method 2 and the advantages of Method 4, some other cases were also chosen for the tests. The common characteristic of these cases is that the upper limits on reactive generations and/or transmission line currents have been reduced for a few hours to force the final solution in the base case to be infeasible. This can be done by the following way: first observing the values of the related quantities in the final solution of the base case; then reducing these values by some percentage; finally taking the reduced values as the upper limits. In this way, quite a number of such cases have been constructed and applied to the tests. Among them, four cases which have been presented in Figure 13 are chosen to show the performance of the proposed methods. In the figure, the quantity and its new upper limit for each case are shown. Note that the new upper limit is now equal to the value of that quantity in the final solution of the base case minus the percent reduction given in the figure. The "period" in the figure shows for how long the new limits are effective.

\begin{tabular}{|c|c|c|c|c|}
\hline Case No. & Quantity & Element & $\begin{array}{c}\text { Percent } \\
\text { Reduction }\end{array}$ & Period \\
\hline Case 1 & Current & Line No. 16 & $40 \%$ & 2 hours \\
\hline Case 2 & Current & Line No. 22 & $45 \%$ & 4 hours \\
\hline Case 3 & Current & Line No. 6 & $40 \%$ & 3 hours \\
\hline Case 4 & Current & Line No. 23 & $30 \%$ & 2 hours \\
\hline
\end{tabular}

Figure 13: Descriptions for Cases 1-4 in H-T System B.

Methods 2, 3 and 4 have all been applied to the four cases described in Figure 13. The final solutions given by Method 2 were al detected infeasible by the post-procedure mentioned previously. However, Method 3 and Method 4 have given feasible solutions for all cases. 
According to the tests, Method 4 is shown once more to be the most efficient one. It not only overcomes the deficiency of Method 2 , but also greatly reduced the CPU requirement in comparison to Method 3 .

Figure 14 shows how Method 4 performs compared to Method 3 in the tests from Case 1 to Case 4. By taking Method 3 as standard (i.e. $100 \%$ ), the figure gives the percentages of Method 4 for the error at the final solution, the number of OPF performed and the total CPU requirement.

\begin{tabular}{|c|c|c|c|c|}
\hline Case No. & $\begin{array}{c}\text { Error } \\
\text { at final } \\
\text { solution }^{(*)}\end{array}$ & $\begin{array}{c}\text { No. of } \\
\text { OPF }^{(*)}\end{array}$ & $\begin{array}{c}\text { Total } \\
\text { CPU }^{(*)}\end{array}$ & $\begin{array}{c}\text { Increase on } \\
\text { objective }^{(* *)}\end{array}$ \\
\hline Case 1 & $\approx 0.0$ & $26 \%$ & $29.2 \%$ & $0.5 \%$ \\
\hline Case 2 & $\approx 0.0$ & $23 \%$ & $24.8 \%$ & $0.1 \%$ \\
\hline Case 3 & $\approx 0.0$ & $27 \%$ & $30.2 \%$ & $0.1 \%$ \\
\hline Case 4 & $0.1 \%$ & $29 \%$ & $28.5 \%$ & $11.2 \%$ \\
\hline
\end{tabular}

(*) Taking results given by Method 3 as standard.

(**) Taking results from base case as standard.

Figure 14: Performances of Method 4 with H-T System B.

Increase on the objective is also given in Figure 14. This increase is presented in percent by taking the result from the base case as standard.

Note that all cases converged within 4 iterations. When Method 4 was applied, the actual CPU time is about $45-60$ minutes for Cases 1,2 and 3 , and about 100 minutes for Case 4.

Regarding the Fibonacci search we have found that 5-7 points are normally sufficient for obtaining the directional minimum, and $k$, the result of the line search, is always less then 0.5 . In all tests, $k$ decreases from iteration to iteration and is close to zero at the end.

\section{CONCLUSION}

A new and efficient method has been described for the solution of the Short-Term Hydro-Thermal Dispatch problem, including OPF as the mathematical model of the thermal subsystem. Based on the proposed STHTD, four different methods, which differ in the degree of details in modeling the thermal system, have been tested and investigated.

The importance of including a detailed model for the thermal subsystem in STHTD has been emphasized and proven by numerical simulations..

In comparison with previous work reported in the literature, the proposed algorithm has the following advantages:

(1) It uses a straightforward line search process to guarantee a better solution for each iteration instead of constructing a hydro tube of the auxiliary problem (Ref. [8]).

(2) It uses a network flow algorithm to solve the hydro subproblem. This yields great savings of CPU time compared to a progressive optimality algorithm (Ref. [9]).

(3) The capability of taking into account any functional and/or security constraints in the thermal subsystem makes it superior to existing algorithms which cannot be applied in cases when transmission line current limits have to be accounted for (Ref. [10]).

It is worthwhile to note that the decomposition scheme in solving the proposed STHTD provides the flexibility for the users to employ their favourite OPF modules.

\section{ACKNOWLEDGEMENT}

The authors acknowledge the financial support for this research by grant from the Natural Science and Engineering Research Council of Canada. Support and encouragement by Drs. P. Kundur, M. El-Kady, D. Frances and Mr. D.Y. Wong from Ontario Hydro are highly appreciated.

\section{APENDIX : ILLUSTRATION OF OSCILLATION}

Let us consider a simplified hydro-thermal problem which involves only one thermal generator and one hydro station with a total time hor- izon of two hours. Also, for simplicity, assume that no water inflows exist during the entire period and head variations are neglected. These assumptions result in a constant discharge-power conversion factor $r$ common for all time intervals. In such a case, power production at each hour is directly proportional to the water discharge at that hour.

If the hydro station is coupled to a reservoir and the starting and ending contents of the reservoir are predefined, the hydro subproblem of such a simplified hydro-thermal problem is to determine how a certain amount of water available is shared between the two hours. This hydro subproblem can be formulated as

$$
\begin{aligned}
\text { Max. } & \lambda_{1}\left(r_{1} q_{1}\right)+\lambda_{2}\left(r_{2} q_{2}\right) \\
\text { s.t. } & q_{1}+q_{2} \leq b \\
& 0 \leq q_{1} \leq q_{1}^{M} \\
& 0 \leq q_{2} \leq q_{2}^{M}
\end{aligned}
$$

where $q_{1}^{M}$ and $q_{2}^{M}$ are the allowable maximum discharges at hour 1 and hour 2 respectively. In (A-1), only one component in turbine discharge has been assumed.

To illustrate the oscillations in performing the hydro-thermal iteration, a two dimensional diagram, spanning $q_{1}$ and $q_{2}$, is given in Figure A-1.

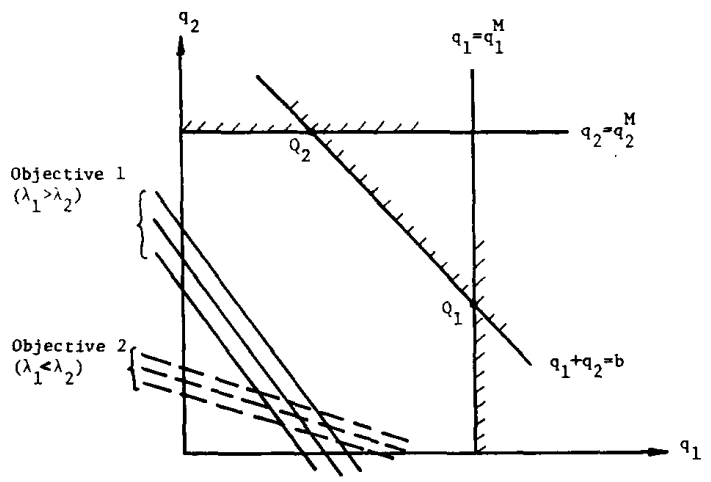

Figure A-1 : Solution process.

Suppose that from the thermal subproblem a relation of $\lambda_{1}>\lambda_{2}$ is given at the present iteration. This relation defines the objective of the hydro subproblem shown by the solid lines in Figure A-1. Accordingly, the solution of problem (A-1) will end up at $Q_{1}$ in the same figure. Point $Q_{1}$ indicates that $q_{1}$ is greater than $q_{2}$ and that $P_{1}$ is greater than $P_{2}$ since $r$ is constant. This result is due to the fact that a certain amount of water has been drawn from hour 2 into hour 1 . To continue the process $P_{1}$ and $P_{2}$ are now fed back to the thermal subproblem. Since $P_{1}$ has been increased and $P_{2}$ has been decreased in comparison to the previous iteration, $\lambda_{1}$ will decrease and $\lambda_{2}$ will increase as a result of the thermal subproblem. This will lead to $\lambda_{1}<\lambda_{2}$. Should this happen, the objective of the hydro subproblem is changed and is now represented by the dashed lines in Figure A-1. Based on this new objective, the solution of the hydro subproblem will end up at $Q_{2}$, which indicates that $P_{1}<P_{2}$ the opposite of the previous iteration. Should the process go on, the solution of the hydro subproblem will jump either from $Q_{1}$ to $Q_{2}$, or from $Q_{2}$ to $Q_{1}$ without convergence.

Even though the iterative process will jump between $Q_{1}$ and $Q_{2}$, one thing certain is however that the solution point must be somewhere in between. Therefore, the solution can be found by a line search along the line segment from $Q_{1}$ to $Q_{2}$, or vice versa. Note that $\lambda_{1}$ and $\lambda_{2}$ used at solution point $Q_{1}$ are the incremental costs (linearization of the hydro-thermal objective). As the solution moves away from $Q_{1}$, this linearization is no longer valid since the incremental costs are changed. Therefore, the variation of the objective must be observed as the solution moves away from $Q_{1}$ to $Q_{2}$ along the line connecting these two points. This will direct the solution towards the optimum and will elliminate the oscillations due to using the incremental costs[20]-[22]. In general, such a line search can efficiently overcome the overshoot and stabilize the solution process. 


\section{References}

[1] E.B. Dahlin and D.W. Shen, "Optimum Solution to the HydroStream Dispatch Problem for Certain Practical Systems", IEEE Trans. Power App. and Syst., vol. PAS-85, 1966, p. 437.

[2] P.L. Dandeno, "Hydrothermal Economic Scheduling - Computational Experience with Co-ordination Equations", AIEE Trans. vol. 80, Feb. 1961, pp. 1219-1228.

[3] H.H. Drake, L.K. Kirchmayer, R.B. Mayall and H. Wood, "Optimal Operation of Hydrothermal Systems", AIEE Trans. Power App. and Syst, vol. PAS-81, 1962, pp. 242.

[4] M.E. El-Hawary and H.G.S. French, "Hydrothermal Dispatch Using Newton-Raphson Method: Theory and Computational Experience", IEEE Winter Power Meeting, Paper A79 046-4, 1979.

[5] H. Brannlund, J.A. Bubenko, D. Sjelvgren and N. Andersson, "Optimal Short Term Operation Planning of a Large HydroThermal Power System Based on a Nonlinear Network Flow Concept", IEEE Winter Power Meeting, Paper A86 050-9, 1986.

[6] H. Habibollahzadeh and J.A. Bubenko, "Application of Decomposition Techniques to Short-Term Operation Planning of Hydrothermal Power System', IEEE Trans. on Power Systems, vol. PWRS-1, no. 1, Feb. 1986, pp. 41-47.

[7] R.A. Duncan, G.E. Seymore, D.L. Streiffert and D.J. Engberg "Optimal Hydrothermal Coordination for Multiple Reservoir River Systems", IEEE Trans. Power App. and Syst., vol. PAS-104, no. 5 , pp. 1154-1159, May 1985.

[8] A.P. Boneart, A.H. El-Abiad and A.J. Koivo, "Optimal Scheduling of Hydro-Thermal Power Systems by Decomposition Technique Using Perturbations", IEEE Trans. Power Appl. and Syst., vol. PAS-91, no. 1, pp. 263-270, 1972.

[9] J. Nanda and P.R. Bijwe, "Optimal Hydrothermal Scheduling with Cascaded Plants Using Progressive Optimality Algorithm", IEEE Trans. Power App. and Syst., vol. PAS-100, no. 4, Apr. 1981, pp. 2093-2099.

[10] M.E. El-Hawary and D.H. Tsang, "The Hydrothermal Optimal Load Flow: A Practical Formulation and Solution Techniques Using Newton's Approach", IEEE Trans. Power Systems., vol. PWRS-1, no. 3, Aug. 1986, pp. 157-167.

[11] J.L. Kennington and R.V. Helgason. Algorithms for Network Programming. John Wiley and Sons, New York, 1981.

[12] M. Minoux, Mathematical Programming Theory and Algorithms, John Wiley and Sons, 1986.

[13] H.H. Happ, "Optimal Power Dispatch -- A Comprehensive Survey", IEEE Trans. Power App. and Syst., vol. PAS-96, no. 3, pp. 841-850, May/June 1977.

[14] H.W. Dommel and W.F. Tinney, "Optimal Power Flow Solutions", IEEE Trans. Power App. and Syst., vol. PAS-87, no. 10, pp. 18661876 , Oct. 1968.

[15] L.K. Kirchmayer, Economic Operation of Power System. John Wiley and Sons, New York, 1958.

[16] G.X. Luo, Short-Term Hydro-Thermal Scheduling Detailed Model and Solutions. Ph.D. Thesis, Dept. of Elec. Engg., University of Toronto, Jan. 1988.
[17] H.H. Happ, R.C. Johnson and W.J. Wright, "Large Scale HydroThermal Unit Commitment - Method and Results", IEEE Trans. Power App. and Syst., vol. PAS-90, no. 3, pp. 1373-1384, May/June 1971.

[18] K. Ea and M. Monti, "Daily Operational Planning of the EDF Plant Mix Proposal for a New Method", IEEE Trans. Power Systems, vol. PWRS-1, no. 3, pp. 251-257, August 1986.

[19] H. Habibollahzadeh, G.X. Luo and A. Semlyen, "Hydrothermal Optimal Power Flow Based on a Combined Linear and Nonlinear Programming Methodology", IEEE Paper no. 88 SM729-6, Presented at the 1988 IEEE/PES Summer Power Meeting at Portland, Oregon.

[20] L.S. Lasdon, Optimization Theory for Large Systems, Macmillan Co., 1970.

[21] M.S. Bazaraa and C.M. Shetty, Nonlinear Programming", John Wiley and Sons, New York, 1979.

[22] G. Zoutendijk, Methods of Feasible Directions, Elsevier Publishing Co., Amsterdam, 1960.

[23] R.E. Rosenthal, "A Nonlinear Network Flow for Maximization of Benefits in a Hydroelectric Power System", Operation Research vol. 29 , no. 4,1981 , pp763-786.

[24] A. Merlin, et al., "Optimization of Short-Term Scheduling of EDF Hydraulic Valley with Coupling Constraints: The OVIDE Model", Proc. of PSCC, Lausane, Switzerland, 1981.

Guang-Xiang Luo was bom in Canton, China on August 22, 1946 He received his B.A.Sc. degree from Wuhan Institute of Hydraulic and Electric Engineering, China, in 1970. From 1970 to 1978, he was employed by Fong-Man Power Station, Northeast China, working at the station control center as a graduate engineer. Since 1980, he has been studying at University of Toronto, Canada, where he received his M.A.Sc. and Ph.D. degrees in electrical engineering in 1983 and 1988, respectively. Recently, he has joined the Ontario Hydro System Planning Division participating in an EPRI project in power system stability.

Hooshang Habibollahzadeh (member of IEEE) was bom in Iran on September 15, 1951. He received his B.S.E.E and M.S.E.E from the University of Washington, Seattle, USA, in 1973 and 1974 respectively. He was a lecturer at the Iran University of Science and Technology, Tehran, Iran, 1975-1981. In 1981 he joined the Energy Systems Laboratory at the Royal Institute of Technology, Stockholm, Sweden, as a research assistant where he received his Ph.D. in 1984. He was a lecturer and researcher at this Institute 1984-1986. He was a research associate at the University of Toronto, Toronto, Canada, 1986-1987. Recently, he has joined the Ontario Hydro Power System Operation Department.

Adam Semlyen (fellow of IEEE) was bom and educated in Rumania where he obtained a Dipl. Ing. degree and his Ph.D. He started his career with an electric power utility and held an academic position at the Polytechnic Institute of Timisoara, Rumania. In 1969 he joined the University of Toronto where he is a professor in the Department of Electrical Engineering. His research interests include the Steady State and Dynamic Analysis of Power Systems, Electromagnetic Transients, and Power System Optimization. 
Discussion

S. K. Tong and S. M. Shahidehpour (Illinois Institute of Technology, IIT Center, Chicago, IL): The authors are to be congratulated for presenting a new algorithm for solving the detailed model of the short-term hydrothermal dispatch problem. We would appreciate the authors' comments on the following issues:

(1) The OPF implemented by the authors for handling the thermal subproblem is based on their previous study [1] which employs a branch-oriented formulation and successive linear programming solution technique. Although this method can provide a more accurate solution than the linear programming method, it demands additional computational resources. Did the authors make comparisons on both efficiency and accuracy between these two methods in their work? The authors have utilized an approximate approach which employs the solution of EOC (an approximate model of OPF) as the solution of the thermal subproblem whenever the solution is feasible. Would it be a good idea to introduce the combination of these two methods which uses the linear programming in the first few iterations to give an approximate solution for the OPF, and applies the successive linear programming technique at the end to achieve more accurate results?

(2) In the description of $C / \lambda$ adjustment, the authors mentioned that the present voltages for the load flow need to be carefully chosen to ensure the feasibility of the solution of EOC. Is there any rigid guideline to set these voltages?

(3) As presented in the paper, the combination of EOC and OPF has led to a great saving in computation time (Figure 14) compared with the case which employs OPF for the entire solution process. This is, of course, attributed to the substitution of the simple EOC for the exact OPF in most of the iterations. In the second example, it has been shown that less than $30 \%$ of the iterations require the exact solution of OPF. Consequently, a $70 \%$ saving in computation time of has been achieved. However, the system that is considered in this paper consists of a smal number of buses. It seems to us that the chance of infeasibility for the solution of EOC increases with the size of the system. Would it also be possible to obtain attractive saving figures for large scale problems?

\section{References}

[1] H. Habibollahzadeh, G. X. Luo, A. Semlyen, "Hydrothermal Optimal Power Flow Based on a Combined Linear and Nonlinear Programming Methodology," IEEE Paper no. 88 SM 729-6, Presented at the 1988 IEEE/PES Summer Power Meeting at Portland, Oregon.

Manuscript received February 16, 1989.

L.A.F.M. FERREIRA (Pacific Gas and Electric Company. 77 Beale St, Room 2455, San Francisco, Ca 94106):

I congratulate the authors for an interesting paper on the subject of hydro-thermal coordination. Their successful development and extensive experimentation with the classical approach of alternately optimizing the thermal and hydro dispatch, using a detailed model for the hydro and an OPF-based model for the thermal system, are most valuable.

In this discussion I would like to make a remark concerning the correctness of the line search procedure proposed in the paper.

The authors propose a piece-wise linear representation for the powerhouse $I / O$ curve, as illustrated in Fig. 2. Then, in the section called $\mathrm{C} \lambda$ Adjustment, they propose (as in [7]) an iterative procedure where each iteration is as follows:

a) select $k \in[0,1]$ (by Fibonacci search)

b) form $\overline{\mathrm{P}}=\overline{\mathrm{P}}_{\mathrm{p}}+\mathbf{k}\left(\overline{\mathrm{P}}_{\mathrm{c}}-\overline{\mathrm{P}}_{\mathrm{p}}\right)$

c) solve the thermal subproblem

where $\overline{\mathrm{P}}_{\mathrm{p}}$ and $\overline{\mathrm{P}}_{\mathrm{c}}$ denote the previous and current hydro productions. The thermal subproblem addresses the load remaining af ter $\overline{\mathbf{P}}$, the new hydro production, has been considered. In this procedure the thermal subproblem is re-solved at every iteration. but the hydro production is assigned new values along the line $\overline{\mathrm{P}}_{\mathrm{P}}$ to $\overline{\mathrm{P}}_{\mathrm{C}}$ according to the value selected for $\mathrm{k}$.

At least in theory, there is a problem of incompatibility between the linear behavior demanded by this procedure and the nonlinear representation of the powerhouse I/O curves. The following example illustrates this.

Consider a hydro system made up of a single reservoir with no other restrictions other than the usage of 2 units of water and a powerhouse whose I/O curve is as indicated in Fig. D. Consider $\overline{\mathrm{p}}_{\mathrm{p}}=\left[\begin{array}{ll}3 & 0\end{array}\right]^{\prime}$ and $\overline{\mathrm{P}}_{\mathrm{c}}=\left[\begin{array}{ll}0 & 3\end{array}\right]^{\prime}$ and $\mathrm{k}=1 / 3$. The new hydro production $\overline{\mathrm{P}}=\left[\begin{array}{ll}2 & 1\end{array}\right]^{\prime}$ does not use 2 units of water, just 1.5 units.

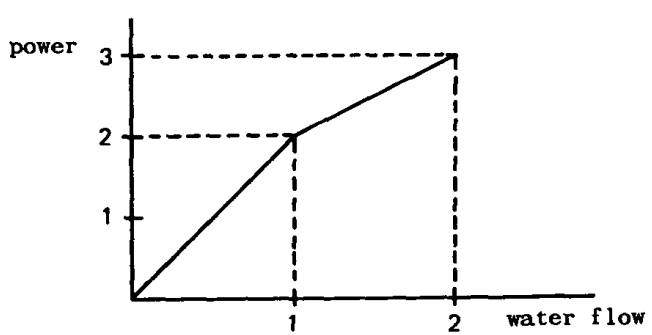

Fig. D

What are the potential practical implications of this problem? Would a linear I/O representation (as used in [7]) be required for realistic applications? It appears that whenever the $1 / 0$ curves are quasi-linear or whenever $\overline{\mathrm{P}}_{\mathrm{p}}$ and $\overline{\mathrm{P}}_{\mathrm{c}}$ are close to each other the practical implications would be minor. In general, however, heuristics are needed to accommodate these mismatches due to nonlinearity. I would appreciate the authors' description of the heuristics used and numerical 11 lustrations concerning this problem.

Manuscript received February 27, 1989.

G.X. Luo, H. Habibollahzadeh, and A. Semlyen: We would like to thank the discussers for their interest in our paper and for their remarks and questions. These give us the opportunity to make the following clarifications.

\section{S.K. Tong and S.M. Shahidehpour:}

(1) The approach used to solve the OPF is the feasible direction technique introduced by Zoutendijk. This method solves an incremental LP to find the feasible direction for the non-linear problem. The solution for this technique could be inside the feasible region because of the line search step and it is not a corner solution. This might not contribute considerable gains in the objective in some cases but it will produce a more practical solution. In a comer solution certain number of variables (nonbasic variables) are forced to be at their bounds which is not favorable in a practical sense. The CPU time used for this technique consists basically of the first LP solution for the feasible direction and the remaining steps and iterations constitute only a small percentage of the CPU time. Therefore an initial LP would not improve the CPU time unless a con- 
ventional model is used for the OPF. This would require two models and corresponding variable conversions between the two models and might not result in any CPU savings.

(2) The load flow used in the $C / \lambda$ Adjustment extends the solution of EOC (the $P$-dispatch of the system) to a full specification of the operating point (the complete $P$ - and $Q$-dispatches of the system). Consequently the feasibility of the solution can be assessed. Normally the load flow result matches closely the $P$-dispatch given by the EOC (except for a small deviation in the slack generation related to the system losses). The $Q$-dispatch and the line flows depend on the voltage settings of the generation buses. Since EOC does not provide any reactive information for the system, proper voltage settings are needed for the load flow extension to obtain a feasible reactive solution. Otherwise, a full scale OPF would be required. Unfortunately, no guidelines are available to set these voltages. Based on experience, in this work the generation voltages were chosen close to their upper bounds. Although this setting works well in our examples, it may be system dependent. However, most of the commercial load flow packages (e.g. the PSS/E load flow or the PECO load flow) have the capability of adjusting the bus voltages and the reactive generations within their bounds. If such a load flow package is employed, this issue becomes less important.
(3) As we stated in our paper, the infeasibility for the solution of EOC depends only on the violation of the reactive generation and line flow limits. The chance of such violations for well designed systems should not vary much with the system size. Therefore it appears that the computational savings shown in our examples will be valid for large scale problems as well.

\section{L.A.F.M. Ferreira:}

We thank the discusser for his careful review of our paper and would like to clarify step (b) of the line search. The inconsistency is due to inappropriately using $P$ instead of $q$ in the equation of step (b). In fact, $q$ is the main variable for the hydraulic problem. Step (b) of the line search is actually a two step process:

(i) use the current and the previous discharges, $q_{c}$ and $q_{p}$, to form the discharge vector $q=q_{p}+k\left(q_{c}-q_{p}\right)$ :

(ii) based on vector $q$, calculate the power vector $P$ according to the I/O curves.

In the discusser's example, $q_{p}=[2,0]^{\prime}$ and $q_{c}=[0,2]^{\prime}$. For $k=1 / 3$ we obtain $q=[4 / 3,2 / 3]^{\prime}$ and $P=[7 / 3,4 / 3]^{\prime}$

Manuscript received April 4, 1989. 